\title{
Artesunate prevents rats from the clozapine- induced hepatic steatosis and elevation in plasma triglycerides
}

This article was published in the following Dove Press journal:

Neuropsychiatric Disease and Treatment

27 September 2017

Number of times this article has been viewed

\author{
Yanmei $\mathrm{Li}^{1,2}$ \\ Ruibing $\mathrm{Su}^{3}$ \\ Shuqin $\mathrm{Xu}^{2}$ \\ Qingjun Huang' \\ Haiyun $\mathrm{Xu}^{1,2}$
}

'The Mental Health Center, Shantou University Medical College, Shantou, Guangdong Province, People's Republic of China; ${ }^{2}$ Department of Anatomy, Shantou University Medical College, Shantou, Guangdong Province, People's Republic of China; ${ }^{3}$ Department of Forensics and Pathology, Shantou University Medical College, Shantou, Guangdong Province, People's Republic of China

\begin{abstract}
Clozapine is an atypical antipsychotic with therapeutic efficacy in treatment-resistant schizophrenia patients and low incidence of extrapyramidal side effects. However, the use of clozapine has been limited by its adverse effects on metabolism. Artesunate is a semisynthetic derivative of artemisinin and was shown to decrease the plasma cholesterol and triglyceride in rabbits and rats in recent studies. The aim of this study was to examine possible effects of artesunate on the clozapine-induced metabolic alterations in rats given saline, clozapine, artesunate, or clozapine plus artesunate for 6 weeks. The clozapine group showed significantly high plasma levels of triglyceride, hepatic steatosis, and fibrosis along with high levels of C-reactive protein, alanine aminotransferase, and aspartate aminotransferase compared to the saline group. But the treatment had no effect on weight gain and caused no hyperglycemia, hyperinsulinemia, and behavioral changes in the rats. More significantly, these clozapine-induced changes were not seen in rats coadministered with clozapine plus artesunate. These results added evidence supporting psychiatrists to try add-on treatment of artesunate in schizophrenia patients to ameliorate clozapine-induced adverse metabolic effects.
\end{abstract}

Keywords: artesunate, clozapine, dyslipidemia, hepatic steatosis, schizophrenia

\section{Introduction}

Clozapine is an atypical antipsychotic distinguished from other antipsychotics by its efficacy in some treatment-resistant patients and its low incidence of extrapyramidal side effects. ${ }^{1-3}$ However, the use of clozapine has been limited by its adverse effects on metabolism in schizophrenia patients, in addition to a risk of agranulocytosis. ${ }^{4}$ The adverse metabolic effects associated with clozapine administration include weight gain, ${ }^{5,6}$ glucose intolerance, ${ }^{5,7,8}$ visceral obesity, ${ }^{9}$ hypertension, ${ }^{10}$ and dyslipidemia. ${ }^{7,11-15}$ These metabolic syndromes may contribute to the increased cardiovascular mortality in schizophrenia patients. Indeed, a recent clinical cohort study with 355 clozapineusing patients reported that older age of commencement with clozapine was associated with increased risk of cardiovascular abnormalities and that an increase in body mass index (BMI) was consistently and significantly associated with all metabolic events. ${ }^{16}$ Moreover, neuroleptic malignant syndrome (NMS) has been a medical emergency in the emergency department, which is associated with the use of antipsychotic drugs, such as typical and atypical antipsychotics including clozapine. ${ }^{17}$ Although it has been generally regarded as an idiosyncratic drug reaction, implying that it is unpredictable and dose-independent, NMS can be described as a complex cascade of dysregulation in multiple neurochemical and neuroendocrine systems, potentially culminating in an
Correspondence: Haiyun Xu The Mental Health Center, Shantou University Medical College, 5I504 I Shantou, Guangdong Province, People's Republic of China Email hyxu@stu.edu.cn 
end-stage hypermetabolic syndrome. ${ }^{18}$ Therefore, research efforts aiming at mitigating these clozapine-induced metabolic disturbances are of paramount importance.

Artesunate is a semisynthetic derivative of artemisinin isolated from the traditional Chinese medicine Artemisia annua L. ${ }^{19}$ Like artemisinin, which exhibits a wide variety of efficacy such as immunosuppressive, anti-inflammatory, antitumor, antiangiogenic, and antiparasitic actions, ${ }^{20-23}$ artesunate showed anti-inflammatory properties in in vitro and in vivo models of rheumatoid arthritis. ${ }^{24-27}$ In a recent study, we found that artesunate decreased expression of the proinflammatory cytokines interleukin-1 $\beta$ (IL-1 $\beta$ ), tumor necrosis factor- $\alpha$ (TNF- $\alpha$ ), and IL-17 $\alpha$ in a rat model of collagen-induced arthritis. ${ }^{28}$ In a more recent study, artesunate attenuated the multiple organ injury and dysfunction of rats submitted to hemorrhage shock, by activating the well-known Akt-survival pathway. ${ }^{29}$ The activation of Akt may result in phosphorylation, and activation of eNOS, thus, enhances the formation of NO, which is pivotal for the preservation of microvascular perfusion, ${ }^{30}$ reducing organ injury. ${ }^{31,32}$ In addition, Akt may turn off the catalytic activity of GSK-3 $\beta,{ }^{33}$ which in turn reduces inflammation. ${ }^{34}$

In addition to its anti-inflammatory properties, artesunate was shown to significantly decrease the plasma cholesterol and triglyceride in rabbits. Furthermore, this drug attenuated liver steatosis and reduced the area of aortic root lesions in the rabbit. ${ }^{35}$ This hypolipidemic effect of artesunate was confirmed in a recent animal study, in which the effects of artesunate on hyperlipidemia were determined in rats fed a Western-type diet that developed a hyperlipidemia. Artesunate alone, or in combination with ursolic acid, prevented hyperlipidemia. Ursolic acid or artesunate alone significantly decreased the plasma triglyceride, but had no effect on the levels of cholesterol. The combination of them, however, reduced both triglyceride and cholesterol, and the effects were more potent than either agent alone. ${ }^{36}$ In line with the above in vivo findings, artesunate inhibited lipid accumulation and triglyceride synthesis without inducing cell toxicity during 3T3-L1 adipocytes differentiation of 8 days and human primary adipocytes differentiation of 20 days. ${ }^{37}$

On the basis of the above literature review, it is rational to hypothesize that artesunate may prevent or mitigate the clozapine-induced metabolic alterations in subjects. To test this hypothesis, this study was performed with adult male Wistar rats treated with clozapine and/or artesunate for 6 weeks. Chronic administration of clozapine was found to increase plasma triglyceride and lead to hepatic steatosis and cirrhosis in the rats. Significantly, these clozapine-induced adverse metabolic effects were not seen in rats coadministered with clozapine and artesunate during the same period.

\section{Materials and methods Drugs}

Clozapine and artesunate were purchased from SigmaAldrich (St Louis, MO, USA). Clozapine was first dissolved in glacial acetic acid, then diluted to its final concentration $(2.0 \mathrm{mg} / \mathrm{mL})$ with sterilized saline, and the $\mathrm{pH}$ value was buffered to 6.0 with sodium hydroxide. Artesunate stock solution was prepared by dissolving it in 5\% sodium bicarbonate, followed by the addition of distilled water. Then the stock solution was stored at $4{ }^{\circ} \mathrm{C}$ until use. The final concentration of artesunate work solution was $0.6 \mathrm{mg} / \mathrm{mL}$ and $\mathrm{pH}=6.0$ adjusted with glacial acetic acid. The drug solutions were freshly prepared every 2 days.

\section{Animals and drug treatments}

A total of 37 adult male Wistar rats were used in this study. The rats were purchased from Beijing Vital River Laboratory Animal Technology Co., Ltd. (Beijing, China). They were housed in groups in the Shantou University Medical College animal facility with free accesses to food and water and a $12 \mathrm{~h}$ dark $/ 12 \mathrm{~h}$ light cycle, at a constant temperature of $24^{\circ} \mathrm{C} \pm 1^{\circ} \mathrm{C}$ and a relative humidity of $60 \%$. All animal procedures applied in this study were in accordance with the guidelines set up by the Animal Care and Use Committee of Shantou University Medical College; the study was approved by the committee.

After acclimation for 7 days, the rats were divided into four groups. In the vehicle control group (termed as saline group; $\mathrm{n}=8$ ), rats were given (intraperitoneal [i.p.]) sterilized saline ( $\mathrm{pH}$ was adjusted to $6.0 ; 0.5 \mathrm{~mL} / 100 \mathrm{~g}$ ) once a day for 6 weeks. In the clozapine group $(n=10)$, rats were given the same volume of clozapine solution ( $\mathrm{pH}=6.0 ; 10 \mathrm{mg} / \mathrm{kg}$, i.p.) once a day during the same period. In the artesunate group $(\mathrm{n}=10)$, rats were given artesunate solution $(\mathrm{pH}=6.0 ; 3 \mathrm{mg} / \mathrm{kg}$, i.p.) once a day during the same period. In the clozapine + artesunate group $(\mathrm{n}=9)$, rats were given clozapine $(10 \mathrm{mg} / \mathrm{kg}$, i.p.) and artesunate (3 mg/kg, i.p.) in the order with a $2-\mathrm{h}$ interval to minimize the possible effect of this larger volume of injection, which was the double of that injected for the other three groups, on the rats. During the experimental period the rats were weighed every week. The doses of artesunate and clozapine used in this study were based on the results of the primary experiment of this study and of our previous studies. ${ }^{28,38,39}$

\section{Behavioral tests}

Twenty-four hours after the last injection, the elevated plus maze, Y-maze, and social interaction tests were performed 
with rats in the order in an interval of $24 \mathrm{~h}$ to evaluate the possible effects of clozapine and artesunate on rats' anxiety level, spatial working memory, and social interaction as described previously. ${ }^{40}$ For elevated plus maze test, a rat was placed at the central junction of the four arms while facing an open arm and allowed to move freely in the maze for 5 min during which period the activity of the rat was recorded. Decreases in time spending on open arms and increases in closed arms were used as a measure of anxietylike behavior. For Y-maze test, each rat was placed at the end of one arm of a symmetrical Y-maze and allowed to move freely through the maze during an 8-min test period. The total number and series of arm entries were recorded. The number of overlapping entrance sequences (eg, ABC, BCA) defines the number of spontaneous alternation. For social interaction test, individual rat was introduced into an open-field $(100 \times 100 \mathrm{~cm})$ and its moving trajectory was tracked for two consecutive sessions of $150 \mathrm{~s}$. During the first session, an empty wire mesh cage $(12 \times 12 \times 18 \mathrm{~cm})$ was placed at one end of the open field. During the second session, the conditions were identical except that a social target animal (an unfamiliar rat of the same gender [male]) had been introduced into the cage. Between the two test sessions, the tested rat was removed from the arena and placed back to its home cage for $\sim 60 \mathrm{~s}$. The video-tracking data from both the no-target and target conditions were used to determine the time spent by the tested rat in the interaction zone (a 16-cm-wide corridor surrounding the cage) and the corners of the open field opposite to the location of the cage using a video tracking program (Noldus Information Technology, Wageningen, the Netherlands).

\section{Biochemical analyses}

For blood sugar test, rats were fasted for $12 \mathrm{~h}$ and blood samples were collected from the tail veins of them. The blood glucose was measured with a blood glucometer (XGP8538TT, Johnson; Shanghai, China). For the measurement of other indices including plasma triglyceride, total cholesterol, alanine aminotransferase (ALT), and aspartate aminotransferase (AST), the rats were deeply anesthetized by sodium pentobarbital $(40 \mathrm{mg} / \mathrm{kg}$ ) and blood was taken by intracardiac puncture and collected in tubes containing $10 \%$ ethylenediaminetetraacetic acid solution. Then the blood sample was centrifuged at $4^{\circ} \mathrm{C}(1,700 \mathrm{rpm})$ for $10 \mathrm{~min}$ to separate blood cells from plasma, which was kept at $-20^{\circ} \mathrm{C}$ until analysis. The measurement of ALT, AST, triglyceride, and total cholesterol levels was performed using the assay kits from Nanjing Jiancheng Biological Engineering Institute (Nanjing, China) and following the instructions provided by the manufacturer. The final values were read by Tecan Microplate Reader (Infinite M200 Pro, Tecan Trading AG, Männedorf, Switzerland).

\section{Enzyme-linked immunosorbent assay}

Insulin, IL-1 $\beta$, TNF- $\alpha$, and C-reactive protein (CRP) of plasma were analyzed by means of enzyme-linked immunosorbent assay method using the assay kit from Mercodia AB (Uppsala, Sweden) for insulin and kits from Boster (Wuhan, China) for the others. At the end, the plates were read by the microplate reader (Bio-Rad laboratories, Inc., CA, USA) at $450 \mathrm{~nm}$.

\section{Histopathological examination}

Under a deep anesthetization, liver tissue samples of rats were collected and kept in 10\% formalin solution for 3 days. Then the tissue samples were embedded with paraffin, followed by sectioning ( $4 \mu \mathrm{m})$ with a Leica microtome (RM2135; Leica, Heidelberg, Germany). The sections were stained by the hematoxylin-eosin (HE) method and examined under a Zeiss microscope (Zeiss Instruments Inc., Göttingen, Germany).

\section{Immunohistochemical staining}

Immunohistochemical staining was performed using the primary antibody against $\alpha$-smooth muscle actin ( $\alpha$-SMA) and an immunohistochemical assay kit (SV0001-mouse IgG) from Boster (Wuhan, China) to examine the activation of hepatic stellate cells and hepatic fibrosis/cirrhosis of rats. Liver specimens were fixed in $10 \%$ neutral buffered formalin and then embedded in paraffin. Liver tissue sections ( $4 \mu \mathrm{m})$ were deparaffinized by xylene and rehydrated in gradient ethanol solutions. After washing in distilled water for $5 \mathrm{~min}$ 3 times, the sections were incubated in $3 \% \mathrm{H}_{2} \mathrm{O}_{2}$ for $10 \mathrm{~min}$ at $22^{\circ} \mathrm{C}$ to block the endogenous enzymes. Following washing in phosphate-buffered saline (PBS), the sections were incubated in the $1.2 \%$ sodium citrate solution under a high-pressure cooking for $3 \mathrm{~min}$ for antigen retrieval. After cooling for $45 \mathrm{~min}$ and washing in PBS (5 $\mathrm{min} \times 3$ ), the sections were incubated in 5\% bovine serum albumin at $22^{\circ} \mathrm{C}$ for $10 \mathrm{~min}$, followed by incubation with the primary antibody to $\alpha$-SMA $(1: 100)$ overnight at $4^{\circ} \mathrm{C}$ and the following incubation at $22^{\circ} \mathrm{C}$ for $45 \mathrm{~min}$. After washing with PBS $(3 \times 5 \mathrm{~min})$, the sections were incubated with the goat antimouse immunoglobulin $\mathrm{G}$-horseradish peroxidase working solution at $37^{\circ} \mathrm{C}$ for $30 \mathrm{~min}$. After washing $3 \times 5 \mathrm{~min}$ in PBS, the immunohistochemical complex was visualized with diaminobenzidine solution at $22^{\circ} \mathrm{C}$ for $8 \mathrm{~min}$. After stopping the reaction and washing in distilled water, the sections were counterstained with hematoxylin, followed by differentiation 
in $1 \%$ hydrochloric alcohol for $3 \mathrm{~s}$. The immunohistochemical controls were performed as above, but with the omission of the primary antibody. Finally, the immunohistochemically stained sections were examined under a Zeiss microscope of Axio Imager A2 (Zeiss instruments Inc.). No positive immunostaining was found in any of the control sections.

\section{Statistical analysis}

All data were expressed as means \pm standard error of the mean and analyzed with the software GraphPad Prism (version 5.01). By considering clozapine and artesunate treatments as two main factors, two-way analysis of variance (ANOVA) was performed followed by one-way ANOVA along with Bonferroni post-tests for all data sets. For body weight of each group, one-way ANOVA was performed followed by Bonferroni post hoc tests, in addition to twoway ANOVA performed for the data of each indicated time point. When a $p$-value was $<0.05$, the difference/effect was considered significant.

\section{Results}

\section{Effects of clozapine and artesunate on behaviors of rats}

In the Y-maze, rats in all four groups (in the order of saline, clozapine, artesunate, and clozapine + Artesunate groups) performed similarly with comparable frequencies of arm visiting (12 $\pm 2,11 \pm 1,11 \pm 1$, and $14 \pm 2$, respectively) and spontaneous alternations $(65.30 \% \pm 1.38 \%, 69.87 \% \pm 4.91 \%$, $66.50 \% \pm 4.02 \%$, and $66.42 \% \pm 4.99 \%$, respectively). Twoway ANOVA revealed no interaction between clozapine and artesunate $\left(F_{(1,33)}=1.63, p=\mathrm{ns}\right.$, for arm visiting frequency; $F_{(1,33)}=1.23, p=$ ns, for spontaneous alternation). Similarly, no differences were found among the four groups in terms of their performances in the elevated plus maze and social interaction tests (not shown).

\section{Artesunate and clozapine showed no effects on the body weight and levels of blood sugar and insulin of rats}

Over the 6-week experimental period, rats in all four groups showed similar weight gain. Even for week 6, two-way ANOVA indicated no interaction between artesunate and clozapine $\left(F_{(1,33)}=0.86, p=\right.$ ns $)$ on the body weight of rats. Oneway ANOVA showed that both clozapine $\left(F_{(1,33)}=2.63, p=\mathrm{ns}\right)$ and artesunate $\left(F_{(1,33)}=0.07, p=\right.$ ns $)$ administrations had no significant effect on the body weight of rats (Figure 1). However, post hoc comparisons revealed significant differences between the groups Artesunate and Clozapine + Artesunate

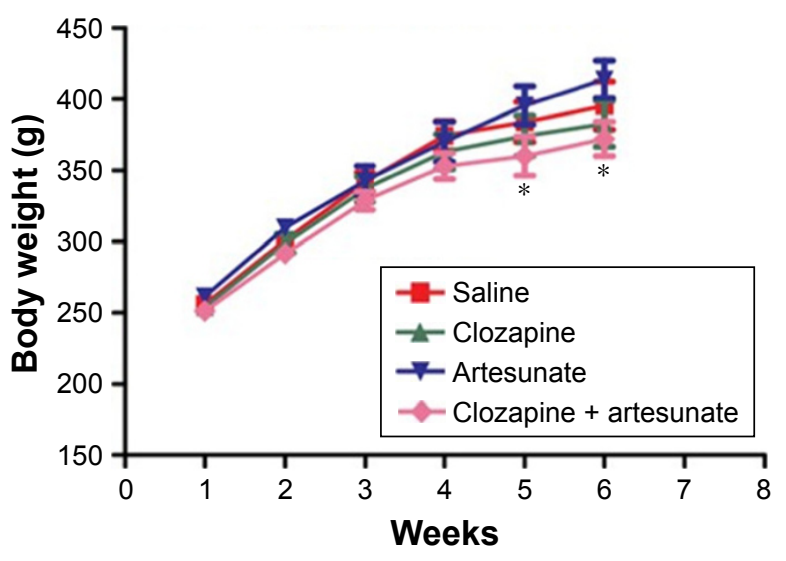

Figure I The weight gain of rats during the 6-week experimental period. Notes: Rats were intraperitoneally given saline, clozapine ( $10 \mathrm{mg} / \mathrm{kg} / \mathrm{day})$, artesunate $(3 \mathrm{mg} / \mathrm{kg} / \mathrm{day})$, or clozapine + artesunate for 6 weeks. Each rat was weighed at the indicated time points. Data were expressed as means \pm standard error of the mean, and analyzed by two-way ANOVA, followed by one-way ANOVA and Bonferroni post-tests. ${ }^{*} p<0.05$, compared to artesunate group.

Abbreviation: ANOVA, analysis of variance.

at the time points of weeks 5 and 6. Two-way ANOVA also indicated no interaction between artesunate and clozapine treatments in terms of the blood $\operatorname{sugar}\left(F_{(1,33)}=1.22, p=\mathrm{ns}\right)$ and insulin $\left(F_{(1,33)}=0.77, p=\right.$ ns $)$ levels. Bonferroni post-tests showed no significant differences between any two groups with respect to blood sugar $(4.1 \pm 0.1,3.7 \pm 0.2,3.8 \pm 0.2$, and $3.8 \pm 0.2 \mathrm{mM} / \mathrm{L}$, respectively) and insulin $(1.732 \pm 0.093$, $1.510 \pm 0.285,1.118 \pm 0.271$, and $1.318 \pm 0.191 \mu \mathrm{g} / \mathrm{L}$, respectively) levels of rats.

\section{Effects of clozapine and artesunate on plasma triglyceride and total cholesterol}

For plasma triglyceride levels of rats, two-way ANOVA indicated no interaction between artesunate and clozapine $\left(F_{(1,33)}=0.42, p=n s\right)$. One-way ANOVA revealed a significant effect of clozapine $\left(F_{(1,33)}=16.14, p<0.001\right)$, but the effect of artesunate was marginal $\left(F_{(1,33)}=2.86, p=0.1\right)$. Bonferroni post-tests showed that clozapine significantly increased levels of plasma triglyceride compared to the saline group (0.9997 $\pm 0.083 \mathrm{mM} / \mathrm{L}$ vs $0.685 \pm 0.0698 \mathrm{mM} / \mathrm{L}, p<0.01)$. But this clozapine-induced increase was not seen in the other two groups (Figure 2A). Interestingly, all four groups showed comparable levels of total cholesterol as shown in Figure 2B.

\section{Artesunate prevents rats from the clozapine-induced hepatic steatosis and fibrosis along with functional impairment}

On the HE-stained tissue sections from all rats $(\mathrm{n}=4-5 /$ group), the lobular organization of liver is evident under the 

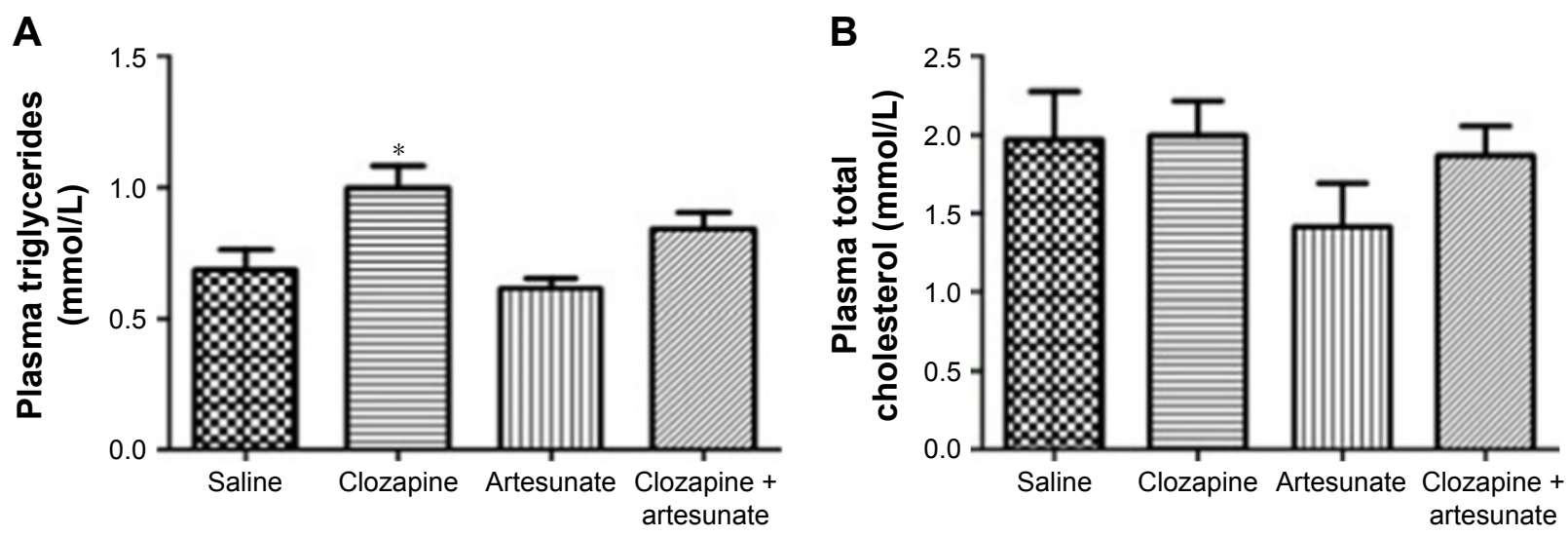

Figure 2 Artesunate prevented rats from the clozapine-induced plasma triglyceride increase.

Notes: Rats were intraperitoneally given saline, clozapine, artesunate, or clozapine + artesunate for 6 weeks. At the end, the rats were deeply anesthetized with sodium pentobarbital, and blood sample was taken by intracardiac puncture and subjected to biochemical analyses for the measurement of plasma triglyceride (A) and total cholesterol (B). Data were expressed as means \pm standard error of the mean, and analyzed by two-way ANOVA, followed by one-way ANOVA and Bonferroni post-tests. $* p<0.05$, compared to saline group.

Abbreviation: ANOVA, analysis of variance.

microscope, characterized with hepatic cords separated by vascular sinusoids connected to the central vein of the liver as shown in Figure 3. However, the typical architecture of hepatic lobules was damaged in liver samples from four of the five rats in the clozapine group; within some of hepatocytes in these rats were empty holes (or vacuoles) where the lipid had been cleared (Figure 3B). No pathological changes were found in liver samples from the other three groups.

The immunohistochemical staining showed evident $\alpha$-SMA immunoreactivity in liver samples from three of the five rats in the clozapine group. The $\alpha$-SMA-positive
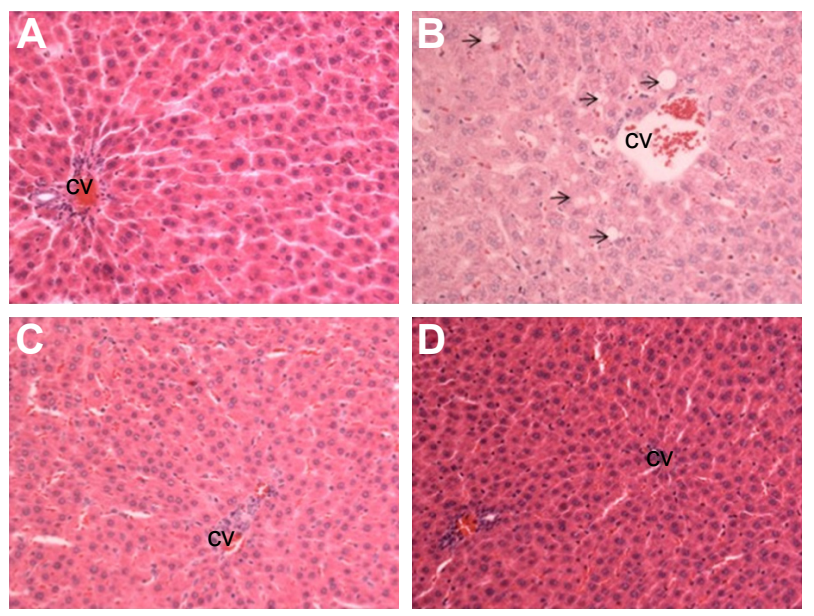

Figure 3 Artesunate prevented rats from the clozapine-induced hepatic steatosis. Notes: Rats were intraperitoneally given saline, clozapine, artesunate, or clozapine + artesunate for 6 weeks. At the end, the rats were deeply anesthetized with sodium pentobarbital and liver sample was taken and processed for hematoxylin-eosin staining. Representative images were from saline group (A), clozapine group (B), artesunate group (C), and clozapine + artesunate group (D) rats. The arrows in image $\mathbf{B}$ point to intracellular vacuoles where the lipid had been cleared. 200× magnification. Abbreviation: $C V$, central vein. cells appeared among hepatocytes; they even outnumbered hepatocytes and converged to form a large focus of fibrosis in the liver. However, these clozapine-induced pathological observations were not seen in the liver tissue of the other three groups, where $\alpha$-SMA immunoreactivity was seen only in the wall of blood vessels (Figure 4).

Along with the hepatic steatosis and fibrosis, clozapinetreated rats also showed higher levels of ALT and AST, compared to saline group. Two-way ANOVA indicated a significant interaction between clozapine and artesunate on plasma levels of ALT of rats $\left(F_{(1,33)}=7.62, p<0.001\right)$.
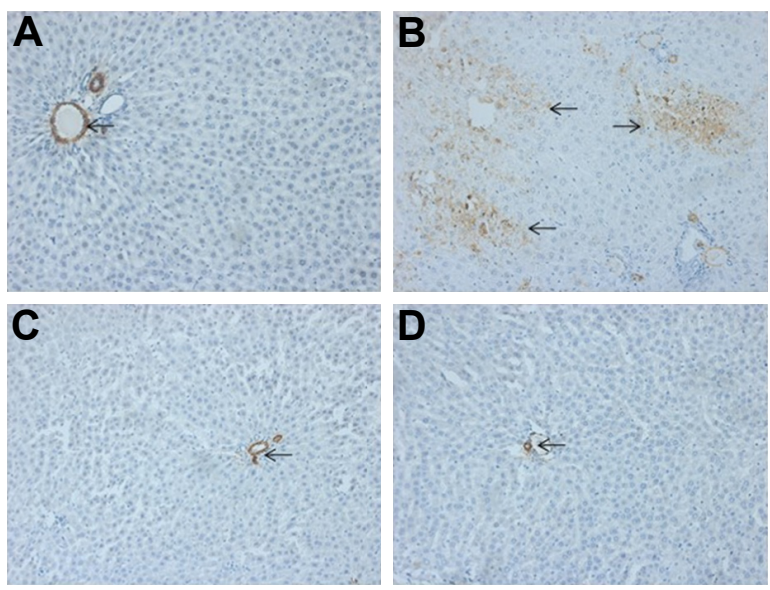

Figure 4 Artesunate prevented rats from the clozapine-induced hepatic fibrosis. Notes: Rats were intraperitoneally given saline, clozapine, artesunate, or clozapine + artesunate for 6 weeks. At the end, the rats were deeply anesthetized with sodium pentobarbital and liver sample was taken and processed for immunohistochemical staining with the primary antibody against $\alpha$-SMA. Representative images were from saline group (A), clozapine group (B), artesunate group (C), and clozapine + artesunate group (D) rats. The arrows in images point to positive $\alpha$-SMA immunostaining. 200 $\times$ magnification.

Abbreviation: $\alpha$-SMA, $\alpha$-smooth muscle actin. 

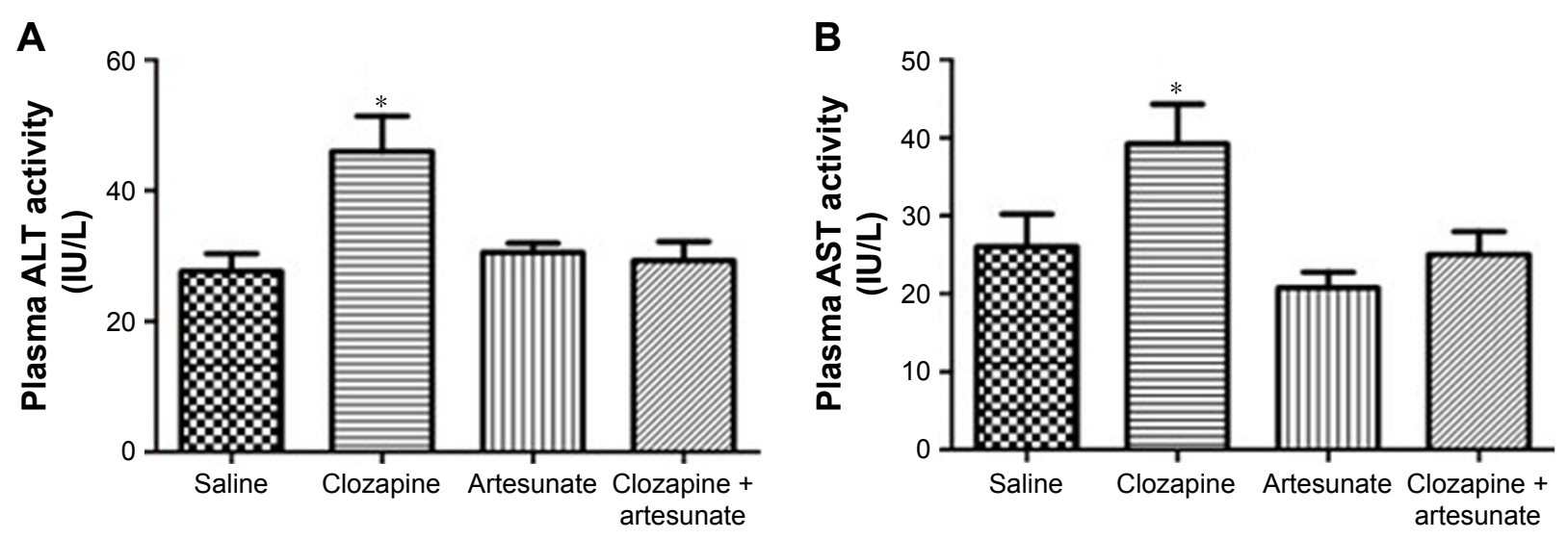

Figure 5 Artesunate prevented rats from the clozapine-induced increases in plasma ALT and AST.

Notes: Rats were intraperitoneally given saline, clozapine, artesunate, or clozapine + artesunate for 6 weeks. At the end, the rats were deeply anesthetized with sodium pentobarbital and blood sample was taken by intracardiac puncture and subjected to biochemical analyses for the measurement of plasma ALT (A) and AST (B). Data were expressed as means \pm standard error of the mean, and analyzed by two-way ANOVA, followed by one-way ANOVA and Bonferroni post-tests. * $p<0.05$, compared to saline group.

Abbreviations: ALT, alanine aminotransferase; ANOVA, analysis of variance; AST, aspartate aminotransferase.

One-way ANOVA showed that both clozapine $\left(F_{(1,33)}=5.82\right.$, $p<0.05)$ and artesunate $\left(F_{(1,33)}=4.74, p<0.05\right)$ showed significant effects. Bonferroni post-tests showed that Clozapine significantly increased levels of ALT in rats compared to the saline group (46.02 $\pm 5.41 \mathrm{IU} / \mathrm{L}$ vs $27.67 \pm 2.72 \mathrm{IU}, p<0.01)$. But this clozapine-induced increase was not seen in the other two groups (Figure 5A). The same statistical conclusions also held true for the AST data of all the four animal groups (Figure 5B).

\section{Effects of clozapine and artesunate on circulating inflammation markers in rats}

Regarding circulating levels of CRP in rats, two-way ANOVA indicated no interaction between artesunate and clozapine $\left(F_{(1,33)}=1.79, p=n s\right)$. One-way ANOVA revealed a significant effect of clozapine $\left(F_{(1,33)}=6.85, p<0.05\right)$, but the effect of artesunate did not reach significant level $\left(F_{(1,33)}=1.76, p=0.1\right)$. Bonferroni post-tests showed that Clozapine significantly increased levels of plasma CRP compared to the saline group $(83.11 \pm 10.27 \mathrm{pg} / \mathrm{mL}$ vs $40.07 \pm 4.60 \mathrm{pg} / \mathrm{mL}, p<0.01)$. But this clozapine-induced increase was not seen in the other two groups (Figure 6A). Interestingly, all four groups showed comparable levels of plasma IL-1 $\beta$ (Figure 6B) and TNF- $\alpha$ (Figure 6C).

\section{Discussion}

As summarized in a recent systematic review and metaanalysis, ${ }^{41}$ metabolic alteration risk was significantly higher with clozapine, followed by olanzapine. The most prevalent manifestations of metabolic alterations in clozapineand olanzapine-treated patients were weight gain, $, 2,43$ hypertriglyceridemia, ${ }^{44}$ and insulin resistance. ${ }^{45}$ In addition, hepatic steatosis ${ }^{46}$ was among the most prevalent risk factors found in patients treated with atypical antipsychotics, especially for those naïve to treatment, in which the drugs caused a more noticeable increase in weight compared with
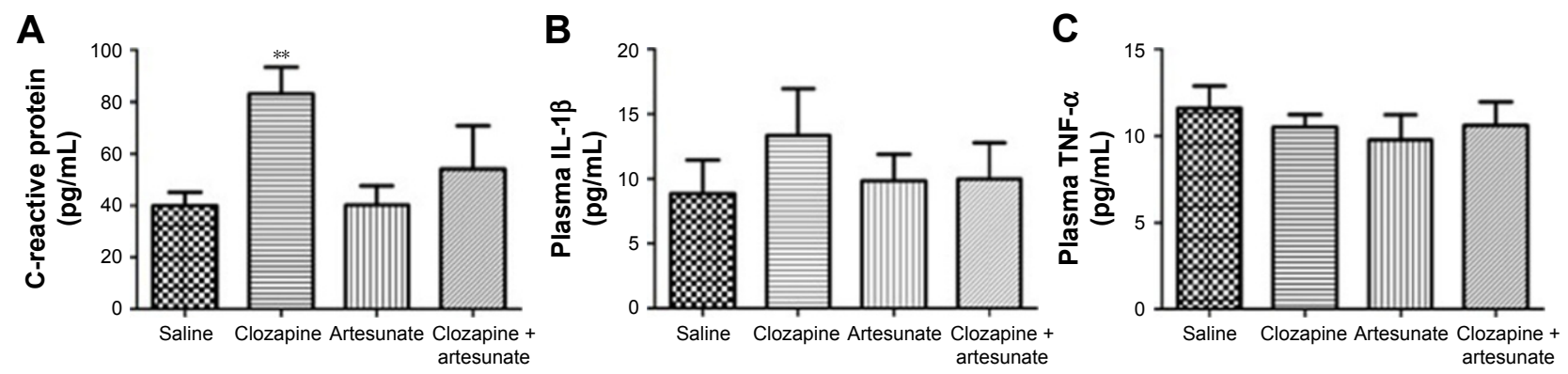

Figure 6 Artesunate prevented rats from the clozapine-induced increase in plasma CRP.

Notes: Rats were intraperitoneally given saline, clozapine, artesunate, or clozapine + artesunate for 6 weeks. At the end, the blood samples of rats were subjected to enzyme-linked immunosorbent assay for the measurement of plasma CRP (A), IL-I $\beta$ (B), and TNF- $\alpha$ (C). Data were expressed as means \pm standard error of the mean, and analyzed by two-way ANOVA, followed by one-way ANOVA and Bonferroni post-tests. $* * p<0.01$, compared to saline group.

Abbreviations: ANOVA, analysis of variance; CRP, C-reactive protein; IL-I $\beta$, interleukin-I $\beta$; TNF- $\alpha$, tumor necrosis factor- $\alpha$. 
chronically treated patients. ${ }^{47}$ Some of these metabolic adverse effects were also mimicked in animal studies. For example, clozapine elevated serum lipid levels in SpragueDawley (S-D) rats, ${ }^{48}$ increased serum triacylglycerol, total cholesterol, free fatty acids, progesterone, and corticosterone levels in the S-D rats, ${ }^{49}$ led to hepatic accumulation of lipids in female S-D rats, ${ }^{50}$ and resulted in hyperglycemia, hyperinsulinemia, and insulin resistance in Wistar albino rats. ${ }^{51}$ In the present study, daily intraperitoneal injection of clozapine for 6 weeks significantly increased plasma levels of triglycerides, led to hepatic steatosis and fibrosis along with increased activities of ALT and AST in male Wistar rats, but showed no effects on blood glucose, insulin levels, and total cholesterol. Some of the clozapine-induced metabolic adverse effects such as increased plasma levels of triglycerides and hepatic steatosis were same as those reported by the above-mentioned previous animal studies, ${ }^{48-50}$ while the others including increased total cholesterol ${ }^{49}$ and hyperglycemia ${ }^{51}$ were not seen in the present study.

In comparing all the animal studies including the present one, we noticed some discrepancies across them. In the study by EI-Seweidy et al, ${ }^{51}$ clozapine was given orally at a dose of $10 \mathrm{mg} / \mathrm{kg}$ body weight daily for 6 weeks and the treatment paradigm resulted in hyperglycemia, hyperinsulinemia, and insulin resistance, whereas the drug was administered by intraperitoneal injection daily for 4 or 6 weeks in the other studies, including the present study and the two studies by Cai et $\mathrm{a}^{49}$ and Dang et $\mathrm{al},{ }^{48}$ all of which showed alterations of dyslipidemia in the absence of changes in glucose metabolism. These discrepancies suggest that the delivery route is an important factor affecting the metabolic side effects of clozapine on subjects. The association of oral administration of clozapine and the concomitant derangement of glucose metabolism in the animal study by EI-Seweidy et $\mathrm{al}^{51}$ helps us understand the insulin resistance and hyperglycemia induced by clozapine in schizophrenia patients who take the drugs orally. ${ }^{5,7,8}$

One of the anthropometric measurements relevant to metabolic alterations induced by atypical antipsychotics is the weight gain in patients. As reviewed in the metaanalysis by Allison et al, ${ }^{52}$ human studies have consistently shown that clozapine and olanzapine are associated with the greatest weight gain. In a recent review, ${ }^{53}$ clozapine and olanzapine were identified as conferring the highest risk of weight gain among atypical antipsychotics, with quetiapine and risperidone having intermediate risk, and aripiprazole and ziprasidone having minimal risk. The meta-analyses in this review value for mean and $95 \% \mathrm{CI}$ of weight change after antipsychotic treatments (up to 12 weeks). Contrary to results of human studies, most of the animal studies showed no weight gain after clozapine treatments; ${ }^{48,51,54-60}$ some of the studies even reported weight loss after clozapine treatment. ${ }^{57,61}$ However, most of the animal studies with olanzapine reported significant weight gain..$^{54,56,62-66}$ This phenomenon seen in animal studies, plus the clinical observation that clozapine induces weight gain in some of schizophrenia patients, $5,7,8$ suggest that the weight gain in schizophrenia patients is more likely due to risk factors for schizophrenia, including genetic factors. For example, variant sites across the IL- $1 \beta$ gene, specifically $r s 16,944 * G A, r s 11,43,634^{*} G$, and $r s 48,49,127^{*} A$, were associated with greater antipsychoticsinduced weight gain in schizophrenia patients. ${ }^{67}$ In addition, the indirect effects of drugs on patients, including inactive lifestyle, length of the disease, ${ }^{68}$ poor dietary choices, and smoking, ${ }^{44}$ may play an important role in the drugs-induced weight gain. In line with the second suggestion, olanzapine was shown to reduce locomotor activity of rats, and this action was significantly correlated with weight gain, suggesting that reduced activity partially contributed to the weight gain induced by olanzapine. ${ }^{63,65,66}$ In the present study, all animal groups displayed similar performances in the Y-maze, elevated plus maze, and social interaction tests, suggesting that each individual drug treatment and combination of clozapine and artesunate had no effect on behaviors of rats, which may explain in part why clozapine treatment paradigm caused no weight gain in this study.

There is a growing body of evidence supporting an important role of cytokine dysfunction in metabolic disturbances such as antipsychotics-induced weight gain in schizophrenia. Specifically, the effects of clozapine and olanzapine on weight gain may be closely related to their effects on cytokine networks as demonstrated in a study by Kluge et al. ${ }^{69}$ In that study, 30 patients diagnosed with schizophrenia, schizophreniform, or schizoaffective disorder were randomized to either clozapine or olanzapine treatment for 6 weeks. Data were longitudinally collected on BMI and plasma levels of leptin and cytokines including TNF- $\alpha$, soluble TNF receptor (sTNFR)-1, sTNFR-2, IL-6, and sIL-2R. All cytokine levels were found to significantly change over time, with the exception of IL- 6 and sTNFR-1 among olanzapine-treated patients. In addition, TNF- $\alpha$, sTNFR-1, sTNFR-2, and sIL-2R levels correlated with BMI after 6 weeks of treatment. These antiinflammatory effects of clozapine were also reported in recent basic studies. One of them showed that clozapine ameliorated the production of microglia-derived TNF- $\alpha$ following the addition of lipopolysaccharide, ${ }^{70}$ while the other one found 
that clozapine administration reversed microglial activation and inducible-nitric-oxide-synthase increase in Wistar rats administered the viral mimetic polyriboinosinic-polyribocytidilic acid (poly I:C). Considering all these previous studies, it is plausible to suggest that the absence of weight gain in clozapine-treated animals may be attributed, at least in part, to its anti-inflammatory action. In line with this suggestion, clozapine treatment paradigm in the present study did not increase plasma levels of cytokines (IL-1 $\beta$ and TNF- $\alpha$ ) and had no effect on weight gain of the rats.

CRP is synthesized in the liver and has been identified as an inflammatory marker. ${ }^{71}$ Increased CRP is associated with multiple risk factors for cardiovascular diseases, including obesity, insulin resistance, and hypertension, and has been demonstrated to have predictive value for risk of metabolic syndrome. ${ }^{72}$ Notably, during treatment with atypical antipsychotics, elevated CRP was associated with high BMI and high glucose levels in patients with psychotic disorders. ${ }^{73}$ There is good evidence that high CRP is associated with increasing serum levels of clozapine. ${ }^{74-79}$ For the first time the present study showed that clozapine administration for 6 weeks increased plasma levels of CRP in rats in the absence of IL- $1 \beta$ and TNF- $\alpha$ changes, suggesting that the increased levels of CRP in these clozapine-treated rats were not due to systemic inflammation. Considering the clozapine-induced hepatic steatosis and fibrosis along with increased ALT and AST, it is conceivable to link the high levels of CRP to liver dysfunction of the rats. In line with this correlation, patients with chronic liver failure had significantly higher CRP levels than others. Remarkably significant differences in CRP levels were still observed between these patients when analyses were restricted to noninfected patients. ${ }^{80}$ Therefore, the block of clozapine-induced CRP increase by artesunate suggests a protective action of this antimalarial on liver functions of the rats. Indeed, co-administration of artesunate and clozapine was shown to prevent rats from the clozapine-induced hepatic steatosis and cirrhosis along with increased plasma levels of triglycerides in the present study. These findings are in accordance with the results of a recent animal study in which artesunate decreased the plasma triglyceride while it attenuated liver steatosis and reduced the area of aortic root lesions in New Zealand rabbits fed a western-type diet developing hyperlipidemia. ${ }^{35}$ These protective effects have specific relevance to the prevention and treatment of the metabolic syndrome including liver dysfunctions seen in some of the schizophrenia patients after chronic administration of clozapine. These are also in line with the beneficial effects of artesunate on the multiple organ injury and dysfunction of rats submitted to hemorrhage shock. ${ }^{29}$
We noticed an interesting phenomenon that both clozapine and artesunate had no effect on the body weight of rats during the 6-week experimental period, whereas significant differences existed between artesunate and clozapine + artesunate groups at weeks 5 and 6 , to wit, the body weight of the latter was lower than that of the former. It seems that both artesunate and the double injection frequency and volume contributed to this difference in opposite directions. The former showed a tendency to increase the body weight of rats, whereas the latter seemed to decrease it. Future studies should avoid this limitation in experimental design. In addition, this study did not explore the underlying molecular mechanisms for the hypolipidemic effects and liver protection of artesunate in clozapine-treated rats. For this goal, in vitro experiments should be done in further studies. Last, futures studies should figure out a safe and effective dose range for the add-on treatment of artesunate and clozapine in small animals, which would provide a meaningful reference for human studies.

In summary, intraperitoneal administration of clozapine for 6 weeks increased plasma levels of triglyceride and lead to hepatic steatosis and cirrhosis along with high levels of CRP, ALT, and AST in rats. But the treatment had no effect on weight gain and caused no hyperglycemia, hyperinsulinemia, and behavioral changes in the rats. These results are in line with most of the previous animal studies associating clozapine injection with hyperlipidemia rather than derangement of glucose metabolism and weight gain, whereas oral administration of clozapine was shown to result in hyperglycemia, hyperinsulinemia, and insulin resistance. These clozapine-induced changes were not seen in rats coadministered with clozapine plus artesunate. Together with recent animal studies ${ }^{35}$ showing hypolipidemic effects of artesunate in New Zealand White rabbits and rats and an in vitro study showing that artesunate inhibited adipogenesis in 3T3-L1 preadipocytes, the hypolipidemic effect of artesunate in clozapine-treated rats shown in the present study provided a significant impetus for psychiatrists to try add-on treatment of this antimalarial in schizophrenia patients with clozapine-induced metabolic adverse effects. If the metabolic side effects of clozapine are effectively blocked by artesunate in schizophrenia patients, the compliance of patients to clozapine will be greatly increased, and, thus, the therapeutic efficacy of it will be significantly improved.

\section{Acknowledgments}

This study was supported by a start-up fund from Shantou University Medical College and a postdoctoral research fellowship from Li Ka Shing Foundation. 


\section{Disclosure}

The authors report no conflicts of interests in this work.

\section{References}

1. Conley RR, Tamminga CA, Kelly DL, Richardson CM. Treatmentresistant schizophrenic patients respond to clozapine after olanzapine non-response. Biol Psychiatry. 1999;46(1):73-77.

2. Freedman R. Schizophrenia. N Engl J Med. 2003;349:1738-1749.

3. Kane J, Honigfeld G, Singer J, Meltzer H. Clozapine for the treatmentresistant schizophrenic. A double-blind comparison with chlorpromazine. Arch Gen Psychiatry. 1988;45(9):789-796.

4. Alvir JM, Lieberman JA. Agranulocytosis: incidence and risk factors. J Clin Psychiatry. 1994;55:137-138.

5. Mookhoek EJ, de Vries WA, Hovens JE, Brouwers JR, Loonen AJ. Risk factors for overweight and diabetes mellitus in residential psychiatric patients. Obes Facts. 2011;4(5):341-345.

6. Umbricht DS, Pollack S, Kane JM. Clozapine and weight gain. J Clin Psychiatry. 1994;55:157-160.

7. Baymiller SP, Ball P, McMahon RP, Buchanan RW. Weight and blood pressure change during clozapine treatment. Clin Neuropharmacol. 2002;25(4):202-206.

8. Wysokinski A. Blood levels of glucose and insulin and insulin resistance in patients with schizophrenia on clozapine monotherapy. Diabetes Metab Syndr. 2014;8(4):236-241.

9. Wysokinski A, Sobow T. Improvements in body composition, anthropometric measurements and lipid profile following discontinuation of clozapine. Nord J Psychiatry. 2016;70(2):156-160.

10. Woo YS, Kim W, Chae JH, Yoon BH, Bahk WM. Blood pressure changes during clozapine or olanzapine treatment in Korean schizophrenic patients. World J Biol Psychiatry. 2009;10(4 Pt 3): $420-425$.

11. Gaulin BD, Markowitz JS, Caley CF, Nesbitt LA, Dufresne RL. Clozapine-associated elevation in serum triglycerides. Am J Psychiatry. 1999;156(8):1270-1272.

12. Ghaeli P, Dufresne RL. Serum triglyceride levels in patients treated with clozapine. Am J Health Syst Pharm. 1996;53(17):2079-2081.

13. Ghaeli P, Dufresne RL. Elevated serum triglycerides with clozapine resolved with risperidone in four patients. Pharmacotherapy. 1999;19(9): 1099-1101.

14. Rettenbacher MA, Ebenbichler C, Hofer A, et al. Early changes of plasma lipids during treatment with atypical antipsychotics. Int Clin Psychopharmacol. 2006;21(6):369-372.

15. Spivak B, Roitman S, Vered Y, et al. Diminished suicidal and aggressive behavior, high plasma norepinephrine levels, and serum triglyceride levels in chronic neuroleptic-resistant schizophrenic patients maintained on clozapine. Clin Neuropharmacol. 1998;21(4):245-250.

16. Hyde N, Dodd S, Venugopal K, Purdie C, Berk M, O’Neil A. Prevalence of cardiovascular and metabolic events in patients prescribed clozapine: a retrospective observational, clinical cohort study. Curr Drug Saf. 2015;10(2):125-131.

17. Belvederi Murri M, Guaglianone A, Bugliani M, et al. Secondgeneration antipsychotics and neuroleptic malignant syndrome: systematic review and case report analysis. Drugs R D. 2015;15(1):45-62.

18. Strawn JR, Keck PE Jr, Caroff SN. Neuroleptic malignant syndrome. Am J Psychiatry. 2007;164:870-876.

19. Liu X. Study on artemisinin derivatives. Yao Xue Tong Bao. 1980;15: 183. Chinese.

20. D'Angelo JG, Bordon C, Posner GH, Yolken R, Jones-Brando L. Artemisinin derivatives inhibit Toxoplasma gondii in vitro at multiple steps in the lytic cycle. J Antimicrob Chemother. 2009;63(1):146-150.

21. Dell'Eva R, Pfeffer U, Vene R, et al. Inhibition of angiogenesis in vivo and growth of Kaposi's sarcoma xenograft tumors by the anti-malarial artesunate. Biochem Pharmacol. 2004;68(12):2359-2366.

22. Hou J, Wang D, Zhang R, Wang H. Experimental therapy of hepatoma with artemisinin and its derivatives: in vitro and in vivo activity, chemosensitization, and mechanisms of action. Clin Cancer Res. 2008; 14(17):5519-5530.
23. Wang JX, Tang W, Shi LP, et al. Investigation of the immunosuppressive activity of artemether on T-cell activation and proliferation. Br J Pharmacol. 2007;150(5):652-661.

24. He Y, Fan J, Lin H, et al. The anti-malaria agent artesunate inhibits expression of vascular endothelial growth factor and hypoxia-inducible factor-1alpha in human rheumatoid arthritis fibroblast-like synoviocyte. Rheumatol Int. 2011;31(1):53-60.

25. Ho WE, Cheng C, Peh HY, et al. Anti-malarial drug artesunate ameliorates oxidative lung damage in experimental allergic asthma. Free Radic Biol Med. 2012;53(3):498-507.

26. Mirshafiey A, Saadat F, Attar M, Di Paola R, Sedaghat R, Cuzzocrea S. Design of a new line in treatment of experimental rheumatoid arthritis by artesunate. Immunopharmacol Immunotoxicol. 2006;28(3): $397-410$.

27. Xu H, He Y, Yang X, et al. Anti-malarial agent artesunate inhibits TNFalpha-induced production of proinflammatory cytokines via inhibition of NF-kappaB and PI3 kinase/Akt signal pathway in human rheumatoid arthritis fibroblast-like synoviocytes. Rheumatology (Oxford). 2007; 46(6):920-926.

28. Li Y, Wang S, Wang Y, et al. Inhibitory effect of the antimalarial agent artesunate on collagen-induced arthritis in rats through nuclear factor kappa B and mitogen-activated protein kinase signaling pathway. Transl Res. 2013;161(2):89-98.

29. Sordi R, Nandra KK, Chiazza F, et al. Artesunate protects against the organ injury and dysfunction induced by severe hemorrhage and resuscitation. Ann Surg. 2017;265(2):408-417.

30. Cabrales $P$, Tsai AG, Intaglietta M. Exogenous nitric oxide induces protection during hemorrhagic shock. Resuscitation. 2009;80(6): $707-712$.

31. Nandra KK, Collino M, Rogazzo M, Fantozzi R, Patel NS, Thiemermann C. Pharmacological preconditioning with erythropoietin attenuates the organ injury and dysfunction induced in a rat model of hemorrhagic shock. Dis Model Mech. 2013;6(3):701-709.

32. Khan AI, Coldewey SM, Patel NS, et al. Erythropoietin attenuates cardiac dysfunction in experimental sepsis in mice via activation of the $\beta$-common receptor. Dis Model Mech. 2013;6(4):1021-1030.

33. Cantley LC. The phosphoinositide 3-kinase pathway. Science. 2002; 296(5573):1655-1657.

34. Martin M, Rehani K, Jope RS, Michalek SM. Toll-like receptormediated cytokine production is differentially regulated by glycogen synthase kinase 3. Nat Immunol. 2005;6(8):777-784.

35. Wang YL, Wang ZJ, Shen HL, Yin M, Tang KX. Effects of artesunate and ursolic acid on hyperlipidemia and its complications in rabbit. Eur J Pharm Sci. 2013;50(3-4):366-371.

36. Yuliang W, Zejian W, Hanlin S, Ming $\mathrm{Y}^{2}$, Kexuan T. The hypolipidemic effect of artesunate and ursolic acid in rats. Pak J Pharm Sci. 2015;28(3):871-874.

37. Jang BC. Artesunate inhibits adipogeneis in 3T3-L1 preadipocytes by reducing the expression and/or phosphorylation levels of C/EBP-alpha, PPAR-gamma, FAS, perilipin A, and STAT-3. Biochem Biophys Res Commun. 2016;474(1):220-225.

38. Xu H, Yang HJ, McConomy B, Browning R, Li XM. Behavioral and neurobiological changes in C57BL/6 mouse exposed to cuprizone: effects of antipsychotics. Front Behav Neurosci. 2010;4:8.

39. Xu H, Yang HJ, Rose GM, Li XM. Recovery of behavioral changes and compromised white matter in C57BL/6 mice exposed to cuprizone: effects of antipsychotic drugs. Front Behav Neurosci. 2011;5:31.

40. Shao Y, Yan G, Xuan Y, et al. Chronic social isolation decreases glutamate and glutamine levels and induces oxidative stress in the rat hippocampus. Behav Brain Res. 2015;282:201-208.

41. Vancampfort D, Stubbs B, Mitchell AJ, et al. Risk of metabolic syndrome and its components in people with schizophrenia and related psychotic disorders, bipolar disorder and major depressive disorder: a systematic review and meta-analysis. World Psychiatry. 2015;14(3): 339-347.

42. Kraus T, Haack M, Schuld A, et al. Body weight and leptin plasma levels during treatment with antipsychotic drugs. Am J Psychiatry. 1999; 156(2):312-314. 
43. Manu P, Dima L, Shulman M, Vancampfort D, De Hert M, Correll CU. Weight gain and obesity in schizophrenia: epidemiology, pathobiology, and management. Acta Psychiatr Scand. 2015;132(2):97-108.

44. Ananth J, Venkatesh R, Burgoyne K, Gadasalli R, Binford R, Gunatilake S. Atypical anti-psychotic induced weight gain: pathophysiology and management. Ann Clin Psychiatry. 2004;16(2):75-85.

45. Lamberti JS, Olson D, Crilly JF, et al. Prevalence of the metabolic syndrome among patients receiving clozapine. Am J Psychiatry. 2006; 163(7):1273-1276.

46. Zhang WV, Ramzan I, Murray M. Impaired microsomal oxidation of the atypical antipsychotic agent clozapine in hepatic steatosis. J Pharmacol Exp Ther. 2007;322(2):770-777.

47. Wani RA, Dar MA, Margoob MA, Rather YH, Haq I, Shah MS. Diabetes mellitus and impaired glucose tolerance in patients with schizophrenia, before and after antipsychotic treatment. J Neurosci Rural Pract. 2015;6(1):17-22.

48. Dang R, Jiang P, Cai H, et al. Vitamin D deficiency exacerbates atypical antipsychotic-induced metabolic side effects in rats: involvement of the INSIG/SREBP pathway. Eur Neuropsychopharmacol. 2015;25(8):1239-1247.

49. Cai HL, Tan QY, Jiang P, et al. A potential mechanism underlying atypical antipsychotics-induced lipid disturbances. Transl Psychiatry. 2015;5:e661.

50. Ferno J, Vik-Mo AO, Jassim G, et al. Acute clozapine exposure in vivo induces lipid accumulation and marked sequential changes in the expression of SREBP, PPAR, and LXR target genes in rat liver. Psychopharmacology (Berl). 2009;203(1):73-84.

51. El-Seweidy MM, Sadik NA, Malek MM, Amin RS. Chronic effects of clozapine administration on insulin resistance in rats: evidence for adverse metabolic effects. Pathol Res Pract. 2014;210(1):5-9.

52. Allison DB, Mentore JL, Heo M, et al. Antipsychotic-induced weight gain: a comprehensive research synthesis. Am J Psychiatry. 1999; 156(11):1686-1696.

53. Lett TA, Wallace TJ, Chowdhury NI, Tiwari AK, Kennedy JL, Müller DJ. Pharmacogenetics of antipsychotic-induced weight gain: review and clinical implications. Mol Psychiatry. 2012;17(3):242-266.

54. Albaugh VL, Henry CR, Bello NT, et al. Hormonal and metabolic effects of olanzapine and clozapine related to body weight in rodents. Obesity (Silver Spring). 2006;14(1):36-51.

55. Cheng CY, Hong CJ, Tsai SJ. Effects of subchronic clozapine administration on serum glucose, cholesterol and triglyceride levels, and body weight in male BALB/c mice. Life Sci. 2005;76(19):2269-2273.

56. Choi S, DiSilvio B, Unangst J, Fernstrom JD. Effect of chronic infusion of olanzapine and clozapine on food intake and body weight gain in male and female rats. Life Sci. 2007;81(12):1024-1030.

57. Cooper GD, Harrold JA, Halford JC, Goudie AJ. Chronic clozapine treatment in female rats does not induce weight gain or metabolic abnormalities but enhances adiposity: implications for animal models of anti-psychotic-induced weight gain. Prog Neuro-psychopharmacol Biol Psychiatry. 2008;32(2):428-436.

58. Hegedus C, Kovacs D, Drimba L, et al. Investigation of the metabolic effects of chronic clozapine treatment on CCK-1 receptor deficient Otsuka Long Evans Tokushima Fatty (OLETF) rats. Eur J Pharmacol. 2013;718(1-3):188-196.

59. Skrede S, Ferno J, Bjorndal B, et al. Lipid-lowering effects of tetradecylthioacetic acid in antipsychotic-exposed, female rats: challenges with long-term treatment. PLoS One. 2012;7(11):e50853.

60. von Wilmsdorff M, Bouvier ML, Henning U, Schmitt A, Gaebel W. The impact of antipsychotic rugs on food intake and body weight and on leptin levels in blood and hypothalamic leptin receptor expression in wistar rats. Clinics (Sao Paulo). 2010;65(9):885-894.
61. Baptista T, Mata A, Teneud L, de Quijada M, Han HW, Hernández L. Effects of long-term administration of clozapine on body weight and food intake in rats. Pharmacol Biochem Behav. 1993;45(1):51-54.

62. Cooper GD, Pickavance LC, Wilding JP, Halford JC, Goudie AJ. A parametric analysis of olanzapine-induced weight gain in female rats. Psychopharmacology. 2005;18(1):80-89.

63. Deng C, Lian J, Pai N, Huang XF. Reducing olanzapine-induced weight gain side effect by using betahistine: a study in the rat model. J Psychopharmacol. 2012;26(9):1271-1279.

64. Lian J, Huang XF, Pai N, Deng C. Preventing olanzapine-induced weight gain using betahistine: a study in a rat model with chronic olanzapine treatment. PLos One. 2014;9(8):e104160.

65. Richards M, Chiba S, Ninomiya M, Wakabayasi C, Kunugi H. Inhibition of olanzapine-induced weight gain by the retinoid analog AM-80. Pharmacopsychiatry. 2013;46(7):267-273.

66. Zhang Q, He M, Deng C, Wang H, Huang XF. Effects of olanzapine on the elevation of macrophage infiltration and pro-inflammatory cytokine expression in female rats. J Psychopharmacol. 2014;28(12): 1161-1169.

67. Fonseka TM, Tiwari AK, Goncalves VF, et al. The role of genetic variation across IL-1beta, IL-2, IL-6, and BDNF in antipsychotic-induced weight gain. World J Biol Psychiatry. 2015;16(1):45-56.

68. Mitchell AJ, Hardy SA. Screening for metabolic risk among patients with severe mental illness and diabetes: a national comparison. Psychiatr Serv. 2013;64(10):1060-1063.

69. Kluge M, Schuld A, Schacht A, et al. Effects of clozapine and olanzapine on cytokine systems are closely linked to weight gain and drug-induced fever. Psychoneuroendocrinology. 2009;34(1):118-128.

70. $\mathrm{Hu} \mathrm{X}$, Zhou $\mathrm{H}$, Zhang D, et al. Clozapine protects dopaminergic neurons from inflammation-induced damage by inhibiting microglial overactivation. J Neuroimmune Pharmacol. 2012;7(1):187-201.

71. Hurlimann J, Thorbecke GJ, Hochwald GM. The liver as the site of C-reactive protein formation. J Exp Med. 1996;123(2):365-378.

72. Shen J, Ordovas JM. Impact of genetic and environmental factors on hsCRP concentrations and response to therapeutic agents. Clin Chem. 2009;55(2):256-264.

73. Dieset I, Hope S, Ueland T, et al. Cardiovascular risk factors during second generation antipsychotic treatment are associated with increased C-reactive protein. Schizophr Res. 2012;140(1-3):169-174.

74. Abou Farha K, van Vliet A, Knegtering H, Bruggeman R. The value of desmethylclozapine and serum CRP in clozapine toxicity: a case report. Case Rep Psychiatry. 2012;2012:592784.

75. Darling P, Huthwaite MA. Infection-associated clozapine toxicity. Clin Schizophr Relat Psychoses. 2011;5(3):159-160.

76. Haack MJ, Bak ML, Beurskens R, Maes M, Stolk LM, Delespaul PA. Toxic rise of clozapine plasma concentrations in relation to inflammation. Eur Neuropsychopharmacol. 2003;13(5):381-385.

77. Hefner G, Shams ME, Unterecker S, Falter T, Hiemke C. Inflammation and psychotropic drugs: the relationship between $\mathrm{C}$-reactive protein and anti-psychotic drug levels. Psychopharmacology (Berl). 2016; 233(9):1695-1705.

78. Pfuhlmann B, Hiemke C, Unterecker S, et al. Toxic clozapine serum levels during inflammatory reactions. J Clin Psychopharmacol. 2009; 29(4):392-394.

79. Raaska K, Raitasuo V, Arstila M, Neuvonen PJ. Bacterial pneumonia can increase serum concentration of clozapine. Eur J Clin Pharmacol. 2002;58(5):321-322.

80. Moreau R, Jalan R, Gines P, et al; CANONIC Study Investigators of the EASL-CLIF Consortium. Acute-on-chronic liver failure is a distinct syndrome that develops in patients with acute decompensation of cirrhosis. Gastroenterology. 2013;144(7):1426-1437. 
Neuropsychiatric Disease and Treatment

Dovepress

\section{Publish your work in this journal}

Neuropsychiatric Disease and Treatment is an international, peerreviewed journal of clinical therapeutics and pharmacology focusing on concise rapid reporting of clinical or pre-clinical studies on a range of neuropsychiatric and neurological disorders. This journa is indexed on PubMed Central, the 'PsycINFO' database and CAS,

and is the official journal of The International Neuropsychiatric Association (INA). The manuscript management system is completely online and includes a very quick and fair peer-review system, which is all easy to use. Visit http://www.dovepress.com/testimonials.php to read real quotes from published authors.

Submit your manuscript here: http://www.dovepress.com/neuropsychiatric-disease-and-treatment-journal 